\title{
Addendum to "Characterising the Strain and Temperature Fields in a Surrogate Bone Material Subject to Power Ultrasonic Excitation"
}

Fabrice Pierron

16 December 2015

\begin{abstract}
Recently, a very interesting article was published in Strain [1] where a rigid polyurethane foam specimen was submitted to longitudinal vibrational excitation in the ultrasonic range. The authors showed that it was possible to measure time resolved strain response maps by combining Digital Image Correlation (DIC) and ultra-high speed imaging. The objective of this discussion is to propose further analysis of the data published in that article, showing that it is possible to extract meaningful values for Young's modulus by using the acceleration field in the specimen as a load cell, as recently proposed in [2]. The aim here is not to provide a complete solution to this problem but to alert the readers on the possibilities offered by this kind of test. This method is an interesting alternative to that presented in [2], where the energy is input repeatedly instead of in one go. Full-field vibration measurements have already been used in the past to identify stiffnesses (see [3-6]) but only in bending and at much lower strain-rates. This article shows that the method can be extended to cover a much wider strain rate range. Finally, only global stiffness values were identified then whereas here, maps of stiffnesses can be derived.
\end{abstract}

\section{Introduction}

The identification of the high strain-rate properties of materials is a very important topic for many engineering applications like crash, blast, forming among others. It is also a very challenging experimental task mainly because of the difficulty in measuring impact loads accurately in regimes where inertia effects are significant. The most popular technique to obtain material parameters at strain-rates between several hundreds to several thousands of $s^{-1}$ is undoubtedly the Kolsky (or Split Hopkinson) Bar. This technique is well understood and produces useful results but suffers from limitations arising from the strong assumptions on which its data analysis relies. In particular, the fact the specimen needs to be at quasi-static equilibrium ( $i e$, forces at both loading ends are equal in magnitude and opposite in sign) is a strong limitations which does 
not allow to obtain data in the transient regime of the test when inertia effects are significant. As a consequence, this technique is not very good at determining elastic stiffness of materials. The present work belongs to a wider effort to invent new high-strain test methodologies based on full-field imaging and inverse identification, to cover up the weaknesses and extend the reach of current techniques like the Split Hopkinson Pressure Bar.

A recent alternative is to use the acceleration information as an imageembedded load cell, where the density of the material acts as the load cell factor, in conjunction with an inverse identification tool called the 'Virtual Fields Method' (VFM), [7]. The first time that this was used was to extract orthotropic stiffness components from vibrating plates [3,4]. This was extended to complex stiffnesses in $[5,6]$. In this case, the assumption of harmonic response makes the calculation of the acceleration much easier as it is simply proportional to the displacements for a given excitation frequency. However, it is possible to extend this approach to transient waves by deriving the acceleration through double temporal differentiation. This was adopted in several papers on composites [2,8], concrete [9] and welds [10]. Recently, a very interesting article was published in Strain [1] where a sawbone specimen was submitted to longitudinal vibrational excitation in the ultrasonic range, and its response measured by Digital Image Correlation (DIC). The objective of the present paper is to show that stiffness data can be extracted from these images using the VFM-based methodology sketched above, which goes one step beyond the analysis performed in the initial paper. This method is an interesting alternative to that presented in [2], where the energy is input repeatedly instead of in one go. Previous work using vibration tests with the VFM [3-6] only addressed bending of thin plates and at much lower strain-rates. This article shows that the method can be extended to cover a much wider strain rate range. Moreover, the present method could readily be extended to study the non-linear stress-strain response of materials, which the work in [3-6] do not cover. Finally, only global stiffness values were identified then whereas here, maps of stiffnesses can be derived. The goal here is not to provide a thorough study for this kind of test but to make the community aware of the potential of this type of test for high rate testing of materials.

\section{Experimental procedure}

The specimen tested here is a rigid polyurethane foam commercially available under the name 'sawbone' (Sawbones Europe, Malmö, Sweden, 0.50 g.cm ${ }^{-3}$, size of $50 \times 10 \times 4 \mathrm{~mm})$. It is excited inertially in the longitudinal direction at a frequency of $20 \mathrm{kHz}$ (see Figure 1), which corresponds to its first resonance frequency. A set of images from [1] was kindly provided by the authors and they were reprocessed by digital image correlation using a different software than in [1]. The images were recorded a few seconds after the start of the test while failure occurred after about $16 \mathrm{~s}$ of test, though the exact moment had not been recorded by the authors of [1]. Figure 1 shows an image of the speckle pattern as recorded by the camera, as per the imaging parameters reported in Table 1. One can see that the high aspect ratio of the specimen strongly limits the lateral spatial resolution. Also, the speckle pattern is rather aliased (ie, small speckles are sampled by less than 4 pixels) which will enhance interpolation bias. One should also be aware of the issues related to the image quality provided 


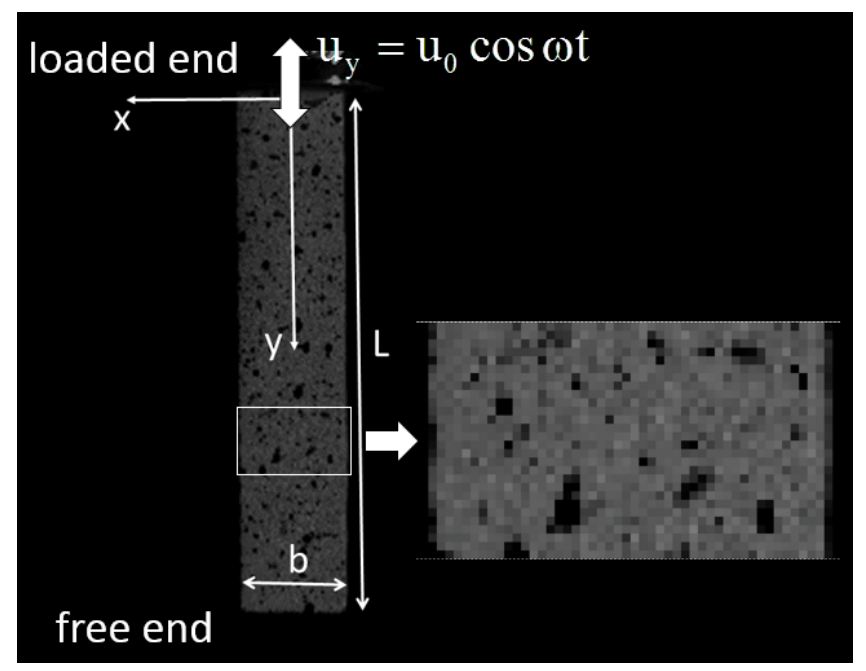

Figure 1: Specimen with speckle pattern, $\mathrm{L}=47.7 \mathrm{~mm}, \mathrm{~b}=10 \mathrm{~mm}$

by the camera, a Shimadzu HPV-1. This camera takes advantage of a specific sensor with data stored onto the chip (In-Situ Image Storage, ISIS). Images are prone to periodical changes in local grey level intensities as reported in detail in [11]. Nevertheless, the images are good enough to extract displacement and strain as shown in [1].

Videos in Section 5 show longitudinal displacement, strain, acceleration and strain-rate maps during the recording which covers about three periods of excitation.

\section{Procedure to identify Young's modulus}

The procedure to identify Young's modulus is based on the analysis presented in [2]. From the principle of virtual work describing the equilibrium of the test specimen, it is possible to reconstruct the average of the longitudinal stress in any given cross-section (at a given position of constant $y$ coordinate) by averaging the longitudinal acceleration over the area between the considered cross-section at position $y$ and the free end. In mathematical terms:

$$
\bar{\sigma}_{y y} y=\rho(L-y) \overline{a_{y}}
$$

where $\rho$ is the density of the specimen, $L$ its length, $y$ the longitudinal coordinate (see Figure 1), $\bar{\sigma}_{y y} y$ is the average of the longitudinal normal stress $\sigma_{y y}$ over a cross-sectional area at position $y$ and $\overline{a_{y}}$ is the surface average of the longitudinal acceleration component $a_{y}$ over the area between the considered cross-section at position $y$ and the free end (area of surface $(L-y) b$ ). This is valid regardless of the constitutive equation as it is pure equilibrium. The only assumption is that the mechanical fields are constant through the thickness since volume integrals appear in the principle of virtual work and only surface measurements are available. It should also be noted that this equation is an approximation as volume integrals are evaluated through discrete data points as provided by 


\begin{tabular}{lc}
\hline Camera & Shimadzu HPV-1 \\
Spatial resolution & $312 \times 260$ \\
Object pixel size & $0.217 \mathrm{~mm}$ \\
Area of interest & $46 \times 220$ pixel $^{2}-10 \times 47.7 \mathrm{~mm}^{2}$ \\
Interframe time & $4 \mu \mathrm{s}$ \\
Shutter speed & $0.5 \mu \mathrm{s}$ \\
Total number of images & 25 \\
\hline Technique used & $2 \mathrm{D}$ DIC \\
Speckle pattern & Paint spray \\
Subset & 21 \\
Shift & 1 \\
\hline Matching criterion & Sum of squared differences \\
Interpolation functions & Bi-cubic splines \\
Shape functions & Affine \\
\hline Displacement & \\
Smoothing method & Linear least square fit \\
Strain window size & 30 data points $($ or pixels $)$ \\
Resolution & Not evaluated \\
\hline Strain & From linear fit \\
Differentiation method & Not evaluated \\
Resolution & Not evaluated \\
\hline Acceleration & Calculation method
\end{tabular}

Table 1: DIC parameters used in the present study 


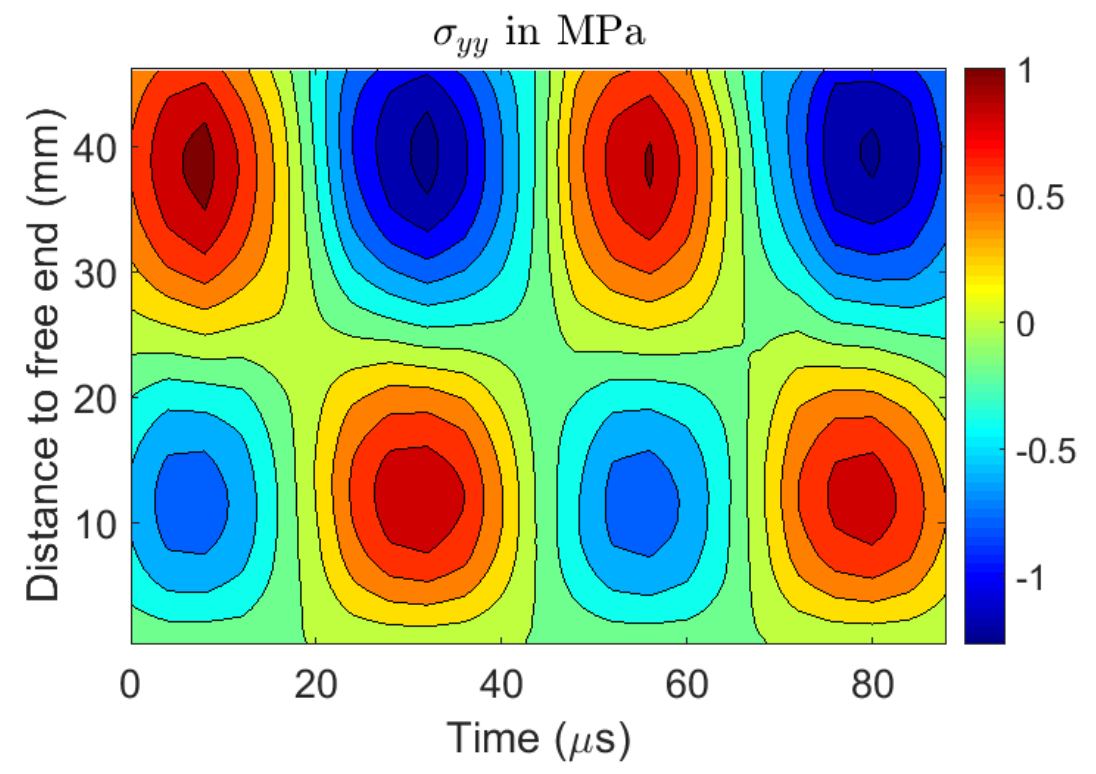

Figure 2: Reconstructed stress ${\overline{\sigma_{y y}}}^{y}$ along the specimen length

DIC. The spatial resolution of the measurements is therefore key. This was not studied here but a route to do this has recently been developed [12] and will be used in future work. Strain is available from the DIC results. It is possible to average the $\varepsilon_{y y}$ strain component in the width $b$ to obtain strain profiles along the specimen length that are consistent with the stress profiles obtained with Equation 1.

\section{Results}

Figure 2 shows stress profiles reconstructed for all time steps and longitudinal positions. One can clearly see that the stress is larger at the top of the specimen. However, plotting the strain profiles (Figure 3), one can see that the strain values do not exhibit such a top to bottom discrepancy. This means that larger Young's modulus values are to be expected in the upper part of the specimen. One can also see that the strain data are much noisier than the acceleration because of the spatial differentiation, whereas the stress data based on acceleration is obtained directly from the displacements, as shown in Table 1. One can also see that the data are less consistent at the top where the maximum is shifted up for the compressive stages. The reason for this is currently unknown.

The strain-rate can also be calculated through:

$$
\dot{\varepsilon}_{y y}=-\omega \varepsilon_{y y}
$$

where $\dot{\varepsilon}_{y y}$ is the longitudinal strain-rate, $\omega$ the angular frequency and $\varepsilon_{y y}$ the longitudinal strain. $\dot{\varepsilon}_{y y}$ is plotted in Figure 4. Because of the harmonic nature of the excitation and the response, the strain-rate map is highly heterogeneous, both in time and space. In future applications of this methodology, the 


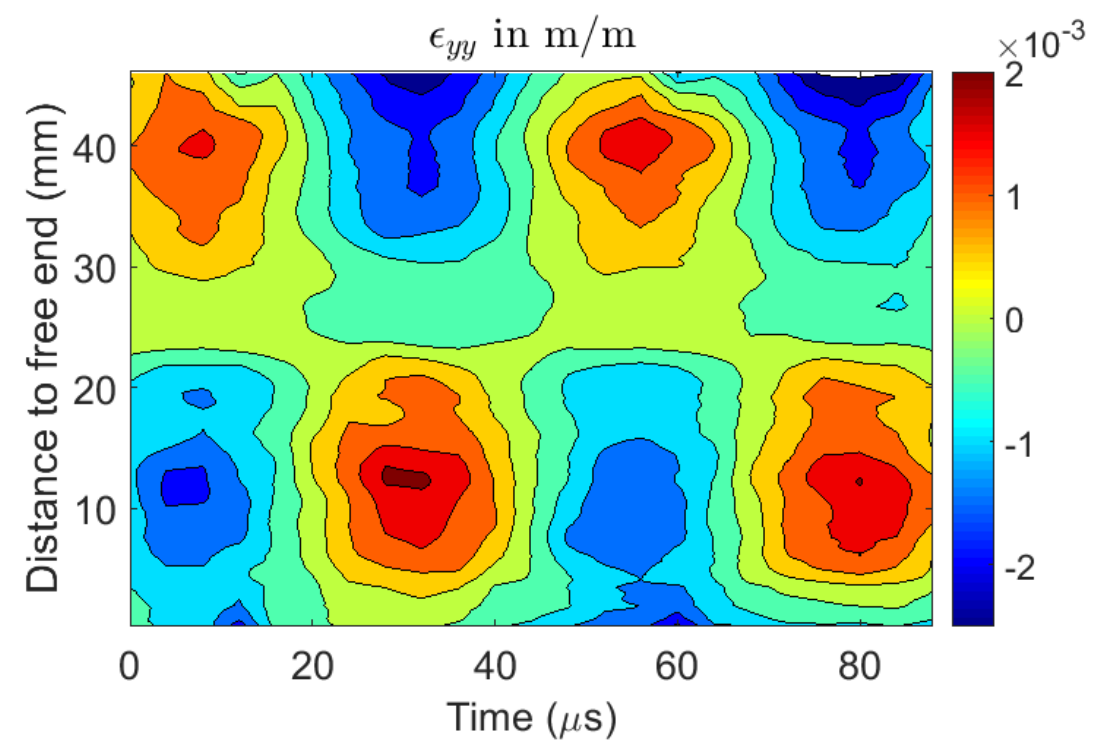

Figure 3: Average $\varepsilon_{y y}$ along the specimen length

strain-rate dependence will have to be explicitly parameterized in the inverse identification procedure.

From the stress and strain data illustrated above, stress-strain curves can be obtained in each cross-section of the specimen. Figure 5 shows two such stressstrain curves for the two locations of maximal stress, at 8.4 and $36 \mathrm{~mm}$ from the top of the specimen. Since about three periods of loading are recorded, three superimposed sets of points make up each stress-strain curve. It can be seen that these curves are reasonably linear, provided the significant strain noise present, caused by limited spatial resolution of the images, and specific imaging issues with this camera [11]. Fitting a straight line to both sets of points, Young's modulus can be calculated. It can clearly be seen that, as expected from the data in Figures 2 and 3, the Young's modulus is significantly larger at the top of the specimen, close to the loaded end. This is confirmed in Figure 6 where the analysis was carried out for all transverse sections and all time steps. The modulus at each time step $t$ is simply obtained by:

$$
E=\sigma_{y y}(t) / \varepsilon_{y y}(t)
$$

where $E$ is Young's modulus. Clearly, there are time steps and locations for which both stress and strain values are too low to provide relevant data. This is the case for locations close to the vibration nodes, and for times where the strain goes to zero between the tensile and compressive parts of the vibration. Therefore, a threshold of $500 \mu d e f$ was set below which the modulus data was not reported. The results are plotted in Figure 6. It can be seen that on average, the moduli values are larger at the top end of the specimen than at the bottom end, as already illustrated above. The data also looks a bit more stable at the bottom end. Generally, the results look consistent along time, if a modulus value is high at one given period at a particular location, this remains the same for the other periods. If averaging values over the top and bottom parts of the specimen, 


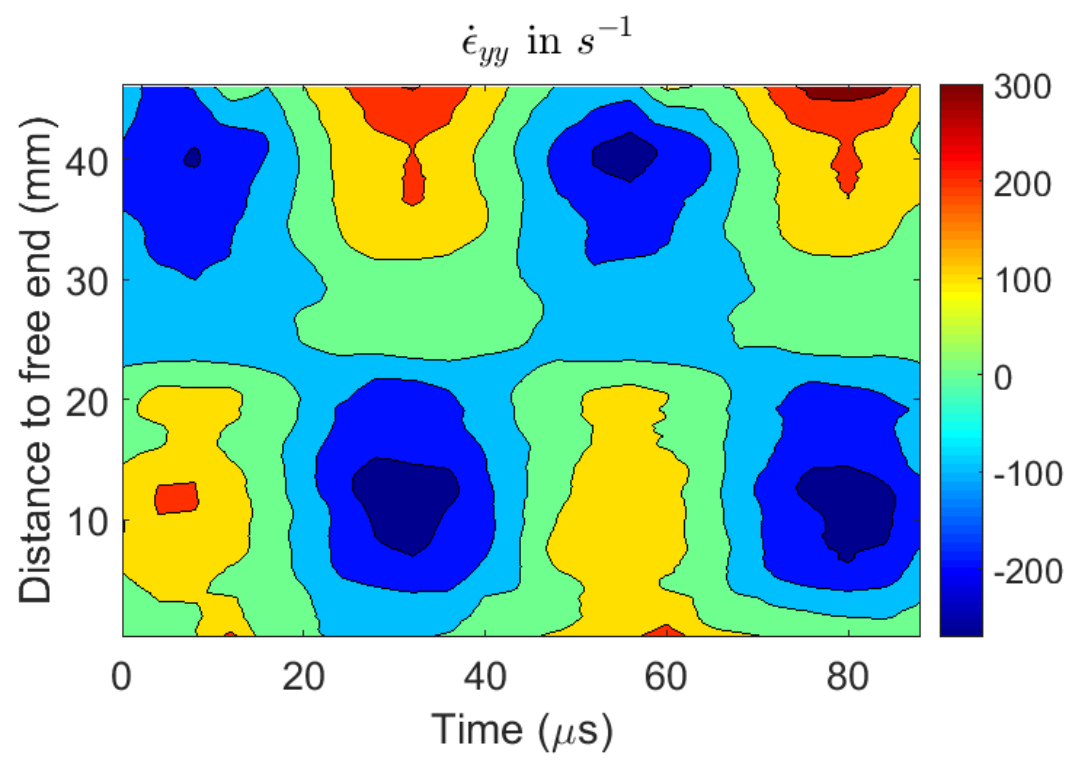

Figure 4: Average $\dot{\varepsilon}_{y y}$ along the specimen length

Young's modulus values of $750 \mathrm{MPa}$ (top) and $450 \mathrm{MPa}$ (bottom) are found. This is to be compared with the $500 \mathrm{MPa}$ value provided by the manufacturer. Although the identified values seem on the whole significantly larger than the datasheet ones, it was shown in previous work [13-15] that the sawbone stiffness datasheet generally reports significantly lower values than reality, most certainly because of inadequate experimental measurement technique. Also, datasheet values are for low rate loading whereas this is at higher rate, hence one could expect a higher modulus value as polyurethane is strain-rate sensitive. There is also the local increase in temperature which would lead to a lowering of the Young's modulus, though looking at Fig. 11 from [1], the temperature is about $60{ }^{\circ} \mathrm{C}$ at the time of image recording, which should not affect the Young's modulus too much as this is still far away from the transition temperature of $120{ }^{\circ} \mathrm{C}$ as illustrated in Fig. 12 of [1]. Finally, sawbone is a foam which is significantly heterogeneous at the local scale so maybe the top to bottom difference relates to local property variations that are not recorded when testing for a global stiffness value using standard tests. In any case, a much more in-depth study based on better and more extensive tests would need to be carried out to get to the bottom of this, but the current values are in-line with expectations. It was also attempted here to obtain the damping factor by fitting each stress and strain data set at each location with a sine function and calculating the phase difference between the two. Unfortunately, the quality of the data is not good enough to yield stable values. However, this will be attempted in the future with better data as this was already successfully done in a more complex case in [6]. 


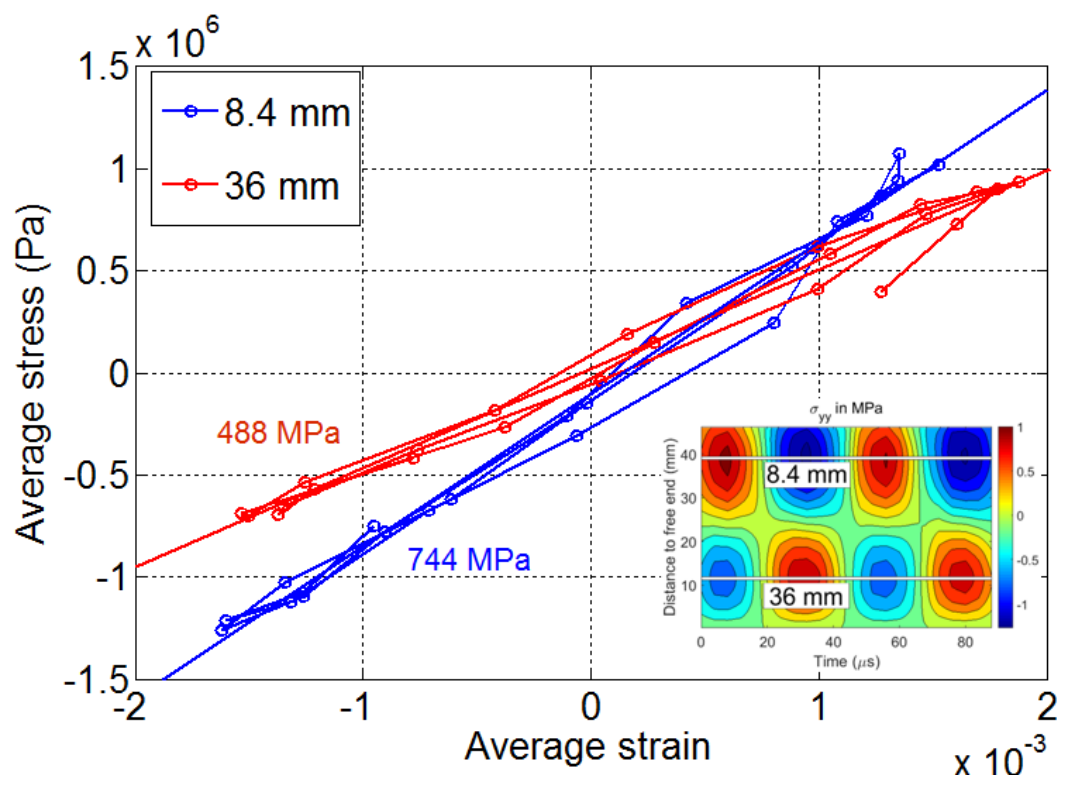

Figure 5: Stress-strain curve for two locations in the sample

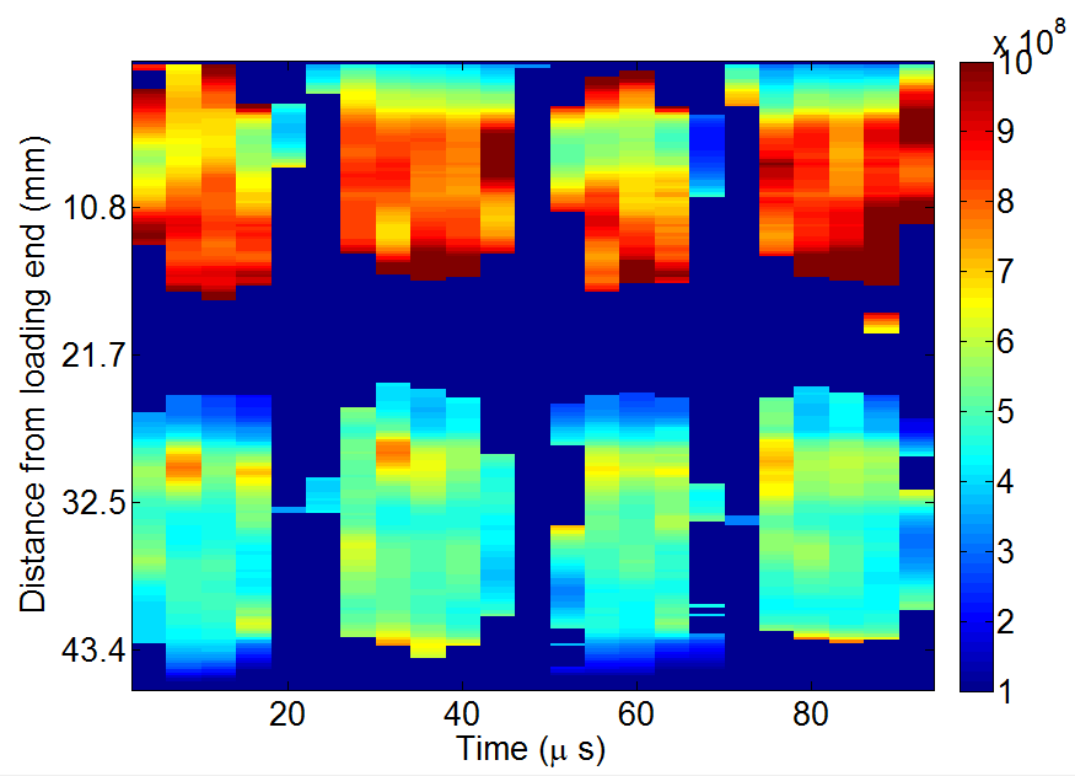

Figure 6: Space-time map of Young's modulus (in Pa) 


\section{Conclusion}

The test proposed in [1] coupled with the analysis described in this article has great potential to explore the strain, strain-rate and temperature dependence of materials constitutive behaviour. [1] also reported temperature measurements. Because of the high excitation frequency and high associated strain rates, selfheating is significant and temperatures reached up to $160{ }^{\circ} \mathrm{C}$ before fracture of the specimen. With better quality measurements, it will be possible to include the identification of temperature dependent stiffness and damping as the test evolves. Therefore, this test has the potential to become an 'imaged-based DMA (Dynamic Mechanical Analysis)' technique. Varying the excitation frequency will enable to shift the high strains from some parts of the specimen to others. This will overcome the problem of the vibration nodes for which no data can be obtained at a particular excitation frequency. Finally, different specimen shapes can also be used to concentrate the strains, or activate more stress components for anisotropic materials.

However, to deliver the above, significant improvement of the measurement quality needs to be achieved. This will be possible by the recent progress in ultra-high speed imaging where better quality images and higher performances are currently at hand $[16,17]$. This is confirmed when comparing the quality of the stress-strain curves obtained in [9] (Fig. 26) with the Shimadzu HPV-1 camera (the same as that used for the present data) and in [2] (Fig. 13) with the more recent HPV-X camera [17]. Improvement will also arise from the use of better speckle patterns with less aliasing, or the use of the grid method when the material allows a grid to be bonded onto it as in [2].

\section{Acknowledgements}

The author is very grateful to Dr Dong Wang and Professor Margaret Lucas for sharing some of the grey level images from [1].

\section{Funding}

The author is thankful to the Royal Society and the Wolfson Foundation for providing him support through a Royal Society Wolfson Research Merit Award. The present work is part of Grant EP/L026910/1 (Established Career Fellowship), for which EPSRC is gratefully acknowledged. Funding from the Air Force Research Laboratory, under agreement number FA9550-15-1-0293 is also acknowledged. The U.S. Government is authorized to reproduce and distribute reprints for Governmental purposes notwithstanding any copyright notation thereon. The views and conclusions contained herein are those of the authors and should not be interpreted as necessarily representing the official policies or endorsements, either expressed or implied, of the Air Force Research Laboratory or the U.S. Government. The authors would like to thank Dr Matt Snyder (EOARD) for supporting this work. 


\section{Supplementary material}

Access to these files is provided on:

http://www.camfit.fr/Supp_Pierron_USwaves_2015.php

- Video of the longitudinal displacement $u_{y}$ in pixel

- Video of the longitudinal strain $\varepsilon_{y y}$

- Video of the longitudinal strain-rate $\dot{\varepsilon}_{y y}$ in $s^{-1}$

- Video of the longitudinal acceleration $a_{y}$ in $m . s^{-2}$

- Grey level images

\section{References}

[1] D. Wang, M. Lucas, and K. E. Tanner, "Characterising the strain and temperature fields in a surrogate bone material subject to power ultrasonic excitation," Strain, vol. 49, no. 5, pp. 409-419, 2013.

[2] F. Pierron, H. Zhu, and C. Siviour, "Beyond Hopkinson's bar," Philosophical Transactions of the Royal Society A: Mathematical, Physical and Engineering Sciences, vol. 372, no. 2023, p. 20130195, 2014.

[3] M. Grédiac and P. A. Paris, "Direct identification of elastic constants of anisotropic plates by modal analysis: Theoretical and numerical aspects," Journal of Sound and Vibration, vol. 195, no. 3, pp. 401-415, 1996.

[4] M. Grédiac, N. Fournier, P. A. Paris, and Y. Surrel, "Direct identification of elastic constants of anisotropic plates by modal analysis: Experimental results," Journal of Sound and Vibration, vol. 210, no. 5, pp. 643-659, 1998.

[5] A. Giraudeau and F. Pierron, "Simultaneous identification of stiffness and damping properties of isotropic materials from forced vibrating plates," Comptes Rendus Mécanique, vol. 331, no. 4, pp. 259-264, 2003.

[6] A. Giraudeau, F. Pierron, and B. Guo, "An alternative to modal analysis for material stiffness and damping identification from vibrating plates," Journal of Sound and Vibration, vol. 329, no. 10, pp. 1653-1672, 2010.

[7] F. Pierron and M. Grédiac, The virtual fields method. Extracting constitutive mechanical parameters from full-field deformation measurements. Springer New-York, 2012.

[8] R. Moulart, F. Pierron, S. R. Hallett, and M. R. Wisnom, "Full-field strain measurement and identification of composites moduli at high strain rate with the virtual fields method," Experimental Mechanics, vol. 51, no. 4, pp. 509-536, 2011.

[9] F. Pierron and P. Forquin, "Ultra high speed full-field deformation measurements on concrete spalling specimens and stiffness identification with the virtual fields method," Strain, vol. 28, no. 5, pp. 388-405, 2012. 
[10] G. Le Louëdec, F. Pierron, M. A. Sutton, C. Siviour, and A. P. Reynolds, "Identification of the dynamic properties of Al $5456 \mathrm{FSW}$ welds using the virtual fields method," Journal of Dynamic Behavior of Materials, vol. 1, no. 2, pp. 176-190, 2015.

[11] M. Rossi, F. Pierron, and P. Forquin, "Assessment of the metrological performance of an in situ storage image sensor ultra-high speed camera for full-field deformation measurements," Measurement Science and Technology, vol. 25, no. 2, p. 025401, 2014.

[12] M. Rossi, P. Lava, F. Pierron, D. Debruyne, and M. Sasso, "Effect of DIC spatial resolution, noise and interpolation error on identification results with the VFM," Strain, vol. 51, no. 3, pp. 206-222, 2015.

[13] T. M. Keaveny, T. P. Pinilla, R. P. Crawford, D. L. Kopperdahl, and A. Lou, "Systematic and random errors in compression testing of trabecular bone," Journal of Orthopaedic Research, vol. 15, no. 1, pp. 101-110, 1997.

[14] A. Marter, F. Pierron, A. Dickinson, and M. Browne, "Traditional methods of strain assessment may under predict cellular foam modulus values," in International Society for Technology in Arthroplasty (ISTA) conference, 2015. 30 September to 3 October 2015 in Vienna, Austria.

[15] A. Marter, F. Pierron, and M. Browne, "Current methods of strain assessment may under predict cellular foam modulus values," Journal of the Mechanical Behaviour of Biomedical Materials, 2015. Submitted.

[16] "Specialized imaging Kirana camera, http://specialisedimaging.com/products/kirana-high-speed-video-camera, last accessed July 30 2015."

[17] "Shimadzu HPV-X camera, http://www.ssi.shimadzu.com/products/product.cfm?product=hpvx_1, last accessed July 30 2015." 